\title{
Monte Carlo simulations of Type I X-ray burst nucleosynthesis
}

\author{
Luke F. Roberts \\ Physics Dept., Colorado College \\ 14 E. Cache La Poudre \\ Colorado Springs, CO 80903, USA
}

\section{W. Raphael Hix}

Physics Division, Oak Ridge National Laboratory

Oak Ridge, TN, 37831-6354, USA

\section{Michael Smith ${ }^{* \dagger}$}

Physics Division, Oak Ridge National Laboratory

Oak Ridge, TN, 37831-6354, USA

E-mail: msmith@mail.phy.ornl.gov

\section{Jacob L. Fisker}

Joint Institute for Nuclear Astrophysics \&

Dept. of Physics, Univ. Notre Dame

225 Nieuwland Hall

Notre Dame, IN 46556-5670, USA

\begin{abstract}
Type I X-ray bursts are recurrent, violent runaway thermonuclear explosions on the surface of an accreting neutron star in a binary star system, driven by reactions on proton-rich unstable nuclei via the rp-process. Large-scale nucleosynthesis calculations are necessary to determine the observed X-ray luminosity and the possible production of rare, low-mass p-nuclides in these outbursts. We have, for the first time, used a Monte Carlo approach with a post-processing nucleosynthesis code to correlate the uncertainties in the (mostly unmeasured) input thermonuclear reaction rates with the uncertainties in the energy generation and synthesized nuclear abundances throughout a burst. Preliminary results and future plans for this study will be discussed.
\end{abstract}

International Symposium on Nuclear Astrophysics - Nuclei in the Cosmos - IX

25-30 June 2006

CERN

\footnotetext{
* Speaker.

${ }^{\dagger}$ ORNL is managed by UT-Battelle, LLC, for the U.S. Department of Energy under contract DE-AC05-00OR22725.
} 


\section{Background and Motivation}

A Type I X-ray burst (XRB) is a violent runaway explosion [1] on the surface of a neutron star caused by a thermonuclear instability in mass that has been transferred from its solar like companion in a binary star system. These outbursts, which can recur hourly, are driven by reactions on proton-rich unstable nuclei via the alpha-p process and rp-process [2,3]. Large-scale nuclear burning calculations are required to determine the energy generation in XRBs that drives the observed X-ray luminosity. Nucleosynthesis calculations can also estimate the possible contribution of these explosions to the abundances of the rare, low-mass p-nuclides such as ${ }^{74} \mathrm{Se},{ }^{78} \mathrm{Kr},{ }^{92,94} \mathrm{Mo}$, and ${ }^{96,98} \mathrm{Ru}$ that are difficult to synthesis in standard p-process scenarios [4].

Roughly a thousand different nuclear reactions are thought to occur in XRBs, and almost all involve proton-rich unstable nuclei and are unmeasured. XRB nucleosynthesis calculations therefore rely heavily on reaction rates derived from statistical model calculations of proton capture cross sections. These cross section estimates, in which the contributions from many closely spaced nuclear levels are averaged, are appropriate for heavier nuclei $(A>40)$ at the high peak temperatures $(\sim 1 \mathrm{GK})$ of XRBs. However, they are not appropriate for reactions through compound nuclei with lower level densities - such as lighter mass nuclei, or heavier nuclei at lower excitation energies that contribute to the rate during the lower temperature cooling phase of the burst [5].

It is therefore important to improve the determination of input thermonuclear rates for XRB simulations via measurements or improved modeling. Because of the large number of reaction rates in XRBs, it is crucial to determine which rates to focus on - specifically, which rates have the largest impact on energy generation (and its related observable, the X-ray luminosity) and on element synthesis. However, because so many reaction rates contribute to the production and destruction of any given nucleus and to the energy generation in a highly coupled way, traditional sensitivity analyses where one or a few reaction rates are varied and the others are held constant cannot uncover all of the correlations between input rates and XRB model predictions. Our study is aimed at developing computation tools - unique for XRB studies - to determine how nuclear reaction rate uncertainties impact understanding of element synthesis and energy generation.

\section{Approach}

Our overall approach involves four parts, the first two of which are completed and published $[6,7,8]$. First, self-consistent 1-dimensional XRB simulations were performed with a code that coupled a full thermonuclear reaction network of 298 isotopes to hydrodynamics including diffusive heat transport, convection, and general relativity $[6,7]$. Second, temperature and density histories of descending Langrangian fluid elements in the envelope were traced in time throughout the burst [8]. In the third part, the current study, post-processing element synthesis calculations were performed with these histories, and a methodology devised to determine the reactions crucial for energy generation and element burning. To do this, we have carried out the first Monte Carlo (MC) element synthesis calculations for an XRB (details below). Finally, the sensitivity of a selfconsistent hydrodynamics plus nuclear burning XRB model to reactions deemed important by the post-processing studies will be investigated in the fourth, future, portion of the project. 
The Monte Carlo approach, first used in Big Bang studies [10,11] and then for novae [12, 13], enables the investigation of the input (i.e., reaction rate) sensitivity of highly-coupled systems with many parameters (i.e., abundances) that are difficult to study with traditional techniques where one or a few inputs are varied while all others are kept fixed. The basic procedure can be summarized as follows. First, we simultaneously and independently vary the value of each input reaction rate for use in a large number of trial simulations. Specifically, we construct the variation of each rate over 50,000 trials to be a log-normal distribution centered on the canonical rate value and with a width corresponding to the rate uncertainty (estimated or determined from experiment). We use the log-normal distribution because nuclear reaction rates are manifestly positive: using a Gaussian or normal distribution of reaction rates can in some cases result in unphysical negative reaction rates.

Then, for each MC trial, we run the post-processing element synthesis code with a temperature and density history from [8] and one of the reaction rate sets. For each trial, we save the rate variations, final abundances, and energy generation in different burst phases. We also determine if the new rates have significantly altered the burst energy generation compared to the canonical run, and therefore violated the self consistency of the post processing calculation; these trials are flagged for further investigation. The distribution of predicted final abundances over the MC trials enables us to quickly determine abundance uncertainties (90\% confidence limits). Figure 1 shows an example of the distribution of how the final abundance predictions for ${ }^{74} \mathrm{Kr}$, a decay precursor to the p-nuclide ${ }^{74} \mathrm{Se}$, are used to determine an abundance uncertainty.

We then determine the correlations of abundance and energy generation with rate variations. To do this, the different energy generation phases of the XRB are first automatically determined by searching for changes in the dominant energy generating reaction in the canonical run. Then, correlations between the input reaction rates and the integration of the energy generation rate over different burst phases are determined. This is followed by determining the correlations between all input reaction rates and the predictions of all final abundances. The real power of the Monte Carlo technique is given by these last two calculations: it is the first time that the impact of simultaneous variation of all input reaction rates has been determined.

\section{Preliminary Results}

- Figure 2 shows the positive correlation between the energy generation rate during the phases leading up to the burst with the rate for the reaction ${ }^{15} \mathrm{O}(\alpha, \gamma){ }^{19} \mathrm{Ne}$. This reaction constitutes a major pathway for processing matter created in the $\mathrm{CNO}$ cycle to higher mass nuclei that can act as seed nuclei for the rp-process [9]. The rate with the most significant impact on energy generation over the entire burst is found to be the triple-alpha reaction rate. The correlation is shown in Figure 3. The rate of the triple-alpha reaction determines if the supply of hydrogen is exhausted in the burst, causing two distinct burning regimes.

- Figure 4 shows the anti-correlation between the abundance of ${ }^{74} \mathrm{Kr}$, a decay precursor to the p-nuclide ${ }^{74} \mathrm{Se}$, and the ${ }^{74} \mathrm{Kr}(\mathrm{p}, \gamma){ }^{75} \mathrm{Rb}$ reaction rate. The sensitivity of abundances to a nearby capture reaction rate is typical throughout our system. The ${ }^{74} \mathrm{Kr}$ abundance is also strongly correlated with the ${ }^{64} \mathrm{Ge}$ decay rate. 


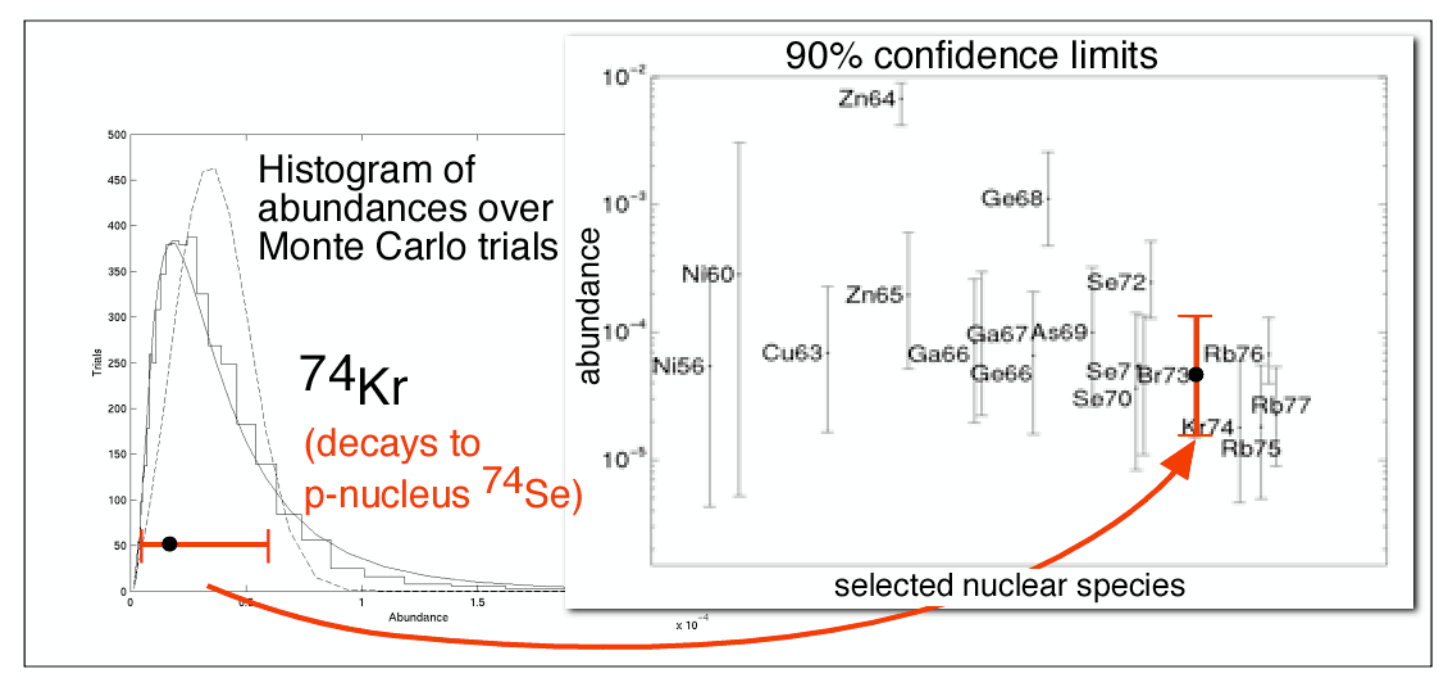

Figure 1: Generation of abundance uncertainties ( $90 \%$ confidence limits) from Monte Carlo trial results

- Some reactions such as ${ }^{23} \mathrm{Al}(\mathrm{p}, \gamma){ }^{24} \mathrm{Si}$, strongly influence the energy generation as well as the final synthesized abundances for a wide range of nuclei; other low-mass proton capture reactions were not found to be nearly as important.

- Decays of ${ }^{24} \mathrm{Si},{ }^{28,30} \mathrm{~S},{ }^{34} \mathrm{Ar},{ }^{38} \mathrm{Ca}$ are strongly correlated with energy generation during different burst phases.

\section{Future Work}

We will repeat our calculations with a new set of improved XRB temperature / density profiles, and then follow this with a detailed study of the sensitivity of a full XRB model with the most important reactions emerging from the Monte Carlo post-processing approach.

\section{References}

[1] S.E. Woosley, R.E. Taam, Nature, 263, 101 (1976).

[2] R.K. Wallace, S.E. Woosley, Astrophys. J. Suppl., 45, 389 (1981).

[3] S.E. Woosley et al., Astrophys. J. Suppl., 151, 75 (2004).

[4] M. Arnould, S. Goriely, Phys. Rept., 384, 1 (2003).

[5] T. Rauscher, F.-K. Thielemann, Phys. Rev. C, 56, 1613 (1997).

[6] J.L. Fisker et al., Astrophys. J., 608, L61 (2004).

[7] J.L. Fisker, E. Brown, M. Liebendorfer, H. Schatz, F.-K. Thielemann, Nucl. Phys. A, 758, 447c (2005).

[8] J.L. Fisker et al., Nucl. Phys. A, 752, 604 (2005).

[9] J.L. Fisker et al., Astrophys. J., in press (2006).

[10] L.M. Krauss, P. Romanelli, Astrophys. J., 358, 47 (1990). 


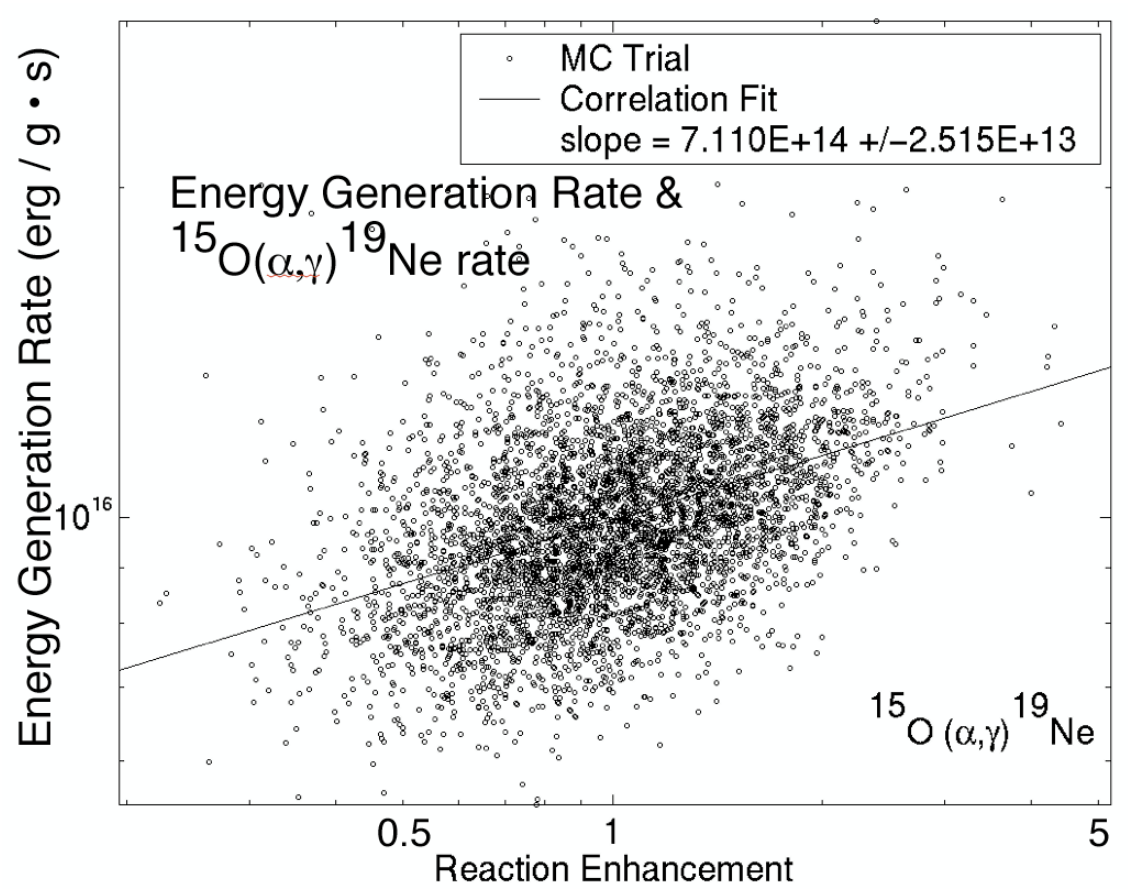

Figure 2: Positive correlation between energy generation phases with the ${ }^{15} \mathrm{O}(\alpha, \gamma){ }^{19} \mathrm{Ne}$ reaction rate

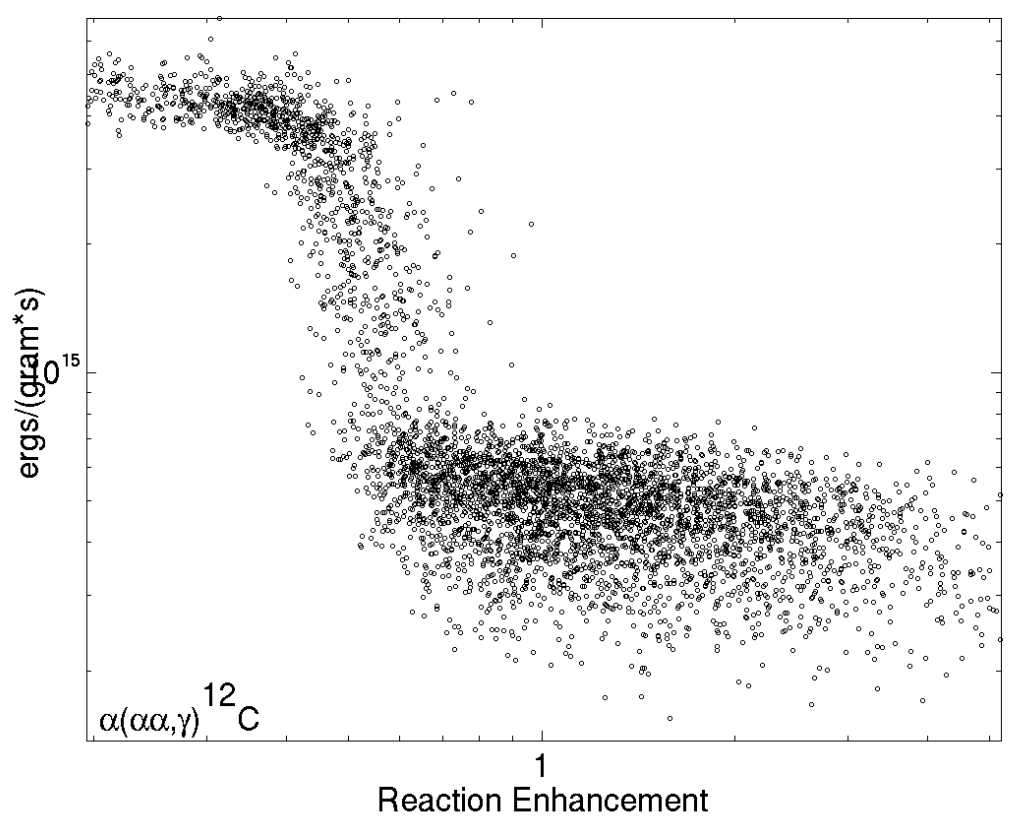

Figure 3: Regimes of hydrogen exhaustion and non-exhaustion caused by variation in the ${ }^{4} \operatorname{He}(2 \alpha, \gamma){ }^{12} \mathrm{C}$ reaction rate 


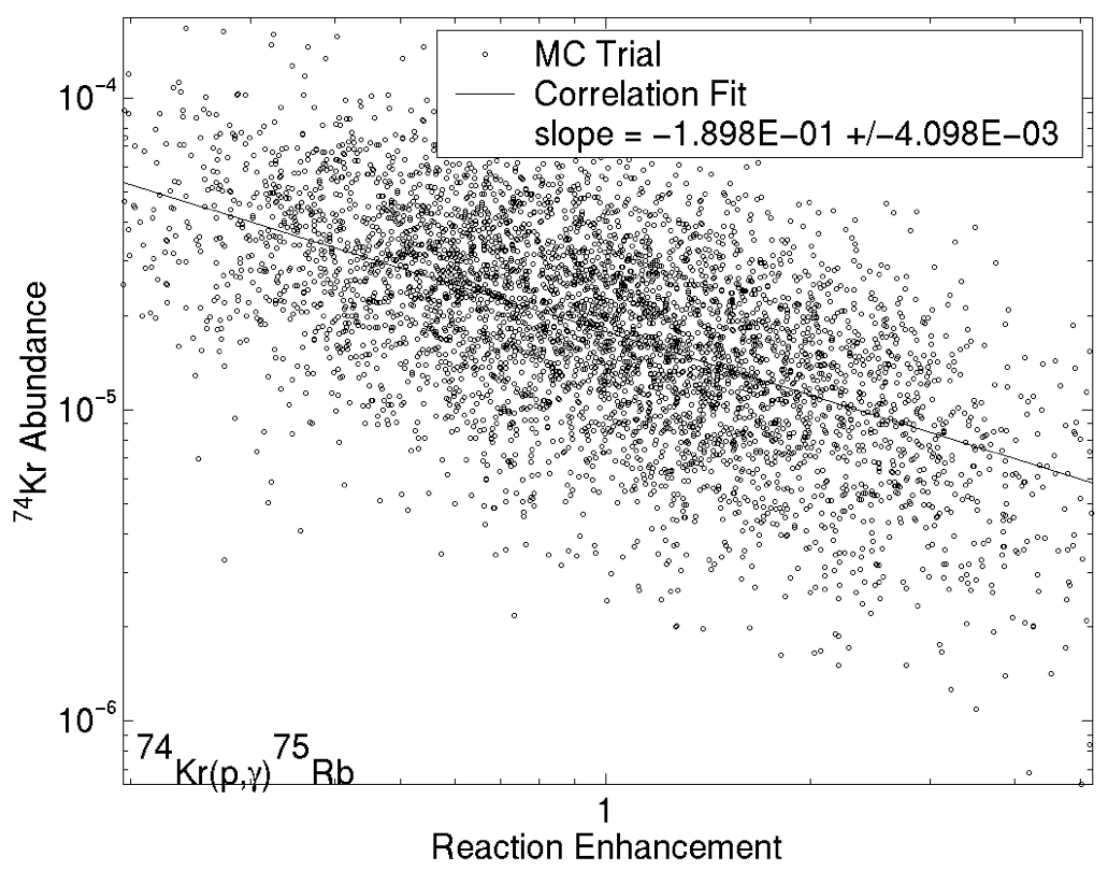

Figure 4: Negative correlation between ${ }^{74} \mathrm{Kr}$ abundance and the ${ }^{74} \mathrm{Kr}(\mathrm{p}, \gamma){ }^{75} \mathrm{Rb}$ reaction rate

[11] M.S. Smith, L.H. Kawano, R.A. Malaney, Astrophys. J. Suppl., 85, 219 (1993).

[12] W.R. Hix, M.S. Smith, A. Mezzacappa, S. Starrfield, D.L. Smith 2003, Nucl. Phys. A 718, 620 (2003).

[13] M.S. Smith et al., in Proc. Int. Conf. on Classical Nova Explosions, eds. M. Hernanz \& J. Jose, (Melville:AIP) 161 (2003). 